\title{
Território e acesso: questões sobre as políticas de saúde brasileiras*
}

\author{
Territory and Access:" Issues about Brazilian Health Policies \\ Territorio y acceso: cuestiones sobre las políticas de salud brasileras
}

\author{
Anita Guazzelli Bernardes** \\ Universidade Católica Dom Bosco
}

Doi: http://dx.doi.org/10.12804/revistas.urosario.edu.co/apl/a.4194

\section{Resumo}

Este artigo tem o objetivo de discutir as articulações entre território e formas de acesso à saúde, a partir da reflexão sobre as Políticas de Saúde brasileiras e o perfil epidemiológico que circunscrevem. Problematiza e coloca em análise alguns elementos para refletir sobre as práticas em saúde, a partir do campo das Políticas de Saúde e do que estas produzem como modos de subjetivação. O texto se apoia em uma perspectiva pós-estruturalista da Psicologia Social, focalizando, principalmente, as ferramentas conceituais foucaultianas. Parte-se da discussão sobre a relação entre Políticas de Saúde e perfil epidemiológico; a partir das considerações dessa relação, articula-se a problemática do território e das formas de acesso. Com base nesses elementos, para finalizar a reflexão, intenta-se apontar algumas questões que nos possibilitam pensar sobre o que o encontro das práticas em saúde com o território produz de diferença. Palavras-chave: território, saúde, perfil epidemiológico, acesso, governamentalidade.
Abstract

This article aims to discuss the articulations between territory and ways of having access to health, from the reflection about brazilian Health Policies and the epidemiological profile they circumscribe. It problematizes and puts under analysis some elements in order to reflect on health practices by considering both the Health Policies field and what they produce as forms of subjectivation. The text is based on a post-structuralist perspective of Social Psychology, focusing mainly on Foucauldian conceptual tools. It begins with the discussion about the relation between Health Policies and epidemiological profile; from the consideration of this relation, the problems of territory and forms of access are articulated. Based on these elements, in order to conclude the reflection, it aims to raise some issues that enable us to think about the difference produced by the encounter of health practices with the territory. Keywords: territory, health, epidemiological profile, access, governmentality.

* Agradeço ao CNPq pelo financiamento da pesquisa da qual se produziu o texto.

** Pós doutorado no Centro de Estudos Sociais da Universidade de Coimbra. Doutora em Psicologia pela Pontifícia Universidade Católica do Rio Grande do Sul. Psicóloga. Docente e Pesquisadora do Programa de Pós-graduação em Psicologia da Universidade Católica Dom Bosco. Pesquisadora do CNPq - bolsista produtividade. Endereço: Rua Av. Tamandaré, 6000 - Jardim Seminário - Campo Grande/MS Cep: 79117-900 - Telefones: 67 - 3312.3300 / 3312.3800 (Programa de Pós-Graduação em Psicologia). Correio electrônico: anitabernardes1909@gmail.com

Cómo citar este artículo: Guazzelli-Bernardes A. (2017). Território e acesso: questões sobre as políticas de saúde brasileiras. Avances en Psicología Latinoamericana, 35(2), 339-349. doi: http://dx.doi.org/10.12804/revistas.urosario.edu.co/apl/a.4194 


\section{Resumen}

Este artículo tiene el objetivo de discutir las articulaciones entre territorio y formas de acceso a la salud, a partir de la reflexión sobre las Políticas de Salud brasileras y el perfil epidemiológico que circunscriben. Problematiza y pone en análisis algunos elementos para reflexionar sobre las prácticas en salud a partir del campo de las Políticas de Salud y de lo que estas producen como modos de subjetivación. El texto se apoya en una perspectiva posestructuralista de la Psicología Social, focalizando principalmente las herramientas conceptuales foucaultianas. Se parte de la discusión sobre la relación entre Políticas de Salud y perfil epidemiológico; a partir de las consideraciones de esa relación, se articula la problemática del territorio y de las formas de acceso. Con base en esos elementos, para finalizar la reflexión, se intenta apuntar algunas cuestiones que nos permiten pensar acerca de lo que el encuentro de las prácticas en salud con el territorio produce de diferente.

Palabras clave: territorio, salud, perfil epidemiológico, acceso, gobernabilidad.

Este artigo tem o objetivo de discutir as articulações entre território e formas de acesso à saúde, a partir da reflexão sobre as Políticas de Saúde e o perfil epidemiológico que circunscrevem. O campo de reflexão são as Políticas de Saúde no Brasil. Esta reflexão parte de um projeto de pesquisa que vem se desenvolvendo desde 2013 e que focaliza as relações entre perfil epidemiológico e políticas públicas de saúde.

O foco na dimensão da articulação entre território e formas de acesso é uma questão que se engendra do processo de investigação das Políticas de Saúde no Brasil e, que neste texto, se trata de uma reflexão teórica sobre esse campo de análise, a partir da Psicologia. Refere-se a uma problematização que põe em análise alguns elementos que permitem refletir sobre as práticas em saúde no Brasil, a partir do campo das Políticas de Saúde e do que estas produzem como modos de subjetivação.
O texto apoia-se em uma perspectiva pós-estruturalista da Psicologia Social, focalizando, principalmente, as ferramentas conceituais foucaultianas. Entende-se que a incorporação dessa perspectiva teórica, no campo da Psicologia, permite outras reflexões, tanto para a disciplina quanto para as políticas públicas. Essas reflexões contribuem para um exercício de pensamento sobre as práticas psicológicas na saúde e sobre as próprias práticas em saúde, na medida em que operam com uma racionalidade em que qualquer prática trata-se de uma política. Ao situar as práticas como políticas, entende-se que se tratam de jogos e tensionamentos entre verdade, poder e subjetividade, a partir dos quais a questão não diz mais respeito a uma perspectiva binária de certo ou errado, mas daquilo que produz em termos de modos de viver.

Nessa perspectiva, a Psicologia neste artigo, se volta para as formas de produção de subjetividades, a partir de distintos vetores de governo das condutas, entre eles a própria saúde. A disciplina entra na esteira de distintas práticas de contestação de modos de assujeitamento, que partem de exercícios de problematização dos diferentes modos de captura e administração da vida e formas de viver.

A proposta é refletir sobre condições de possibilidade que se criam a partir da articulação entre território e formas de acesso como um problema do presente, no sentido de produzir outras grades de inteligibilidade que permitam pensar as Políticas de Saúde. Trata-se, então, de considerar tanto aquilo que, por meio da circunscrição de um perfil epidemiológico, entra em jogo nas relações de governo da vida, quanto o que, ao ficar de fora de um perfil epidemiológico, acaba por engendrar outras possibilidades de vida. Tem-se, portanto, um ensaio teórico que se produz pelo exercício de uma investigação.

Em um primeiro momento, será discutida a relação entre Políticas de Saúde e perfil epidemiológico; a partir das considerações dessa relação, será feita uma articulação com a problemática do território e das formas de acesso. Com base nesses 
elementos, intenta-se, para finalizar a reflexão, apontar algumas questões sobre as quais se torna possível também pensar sobre o que o encontro das práticas em saúde com o território produz de diferença.

\section{Políticas de saúde e perfil epidemiológico}

O perfil epidemiológico é um conceito operacional das Políticas de Saúde e, nesta análise, é tomado como uma estratégia de governamentalidade (Foucault, 2008). É um conceito operacional das Políticas de Saúde, na medida em que por meio dele se organizam programas, metas, focos de promoção, proteção e prevenção em saúde. Como conceito operacional, o perfil epidemiológico permite ao mesmo tempo um conhecimento mais preciso da população e o direcionamento desse conhecimento, em termos de focos de atenção à saúde. Desse modo, traçar um perfil epidemiológico é circunscrever um espaço de ações voltadas para certas características epidêmicas e endêmicas de uma população.

Segundo Czeresnia (2001), a epidemiologia constituir-se-á, sobretudo, a partir do século XVII como uma possibilidade de aproximação entre as dimensões epidêmicas, geográficas, históricas e sociológicas, o que encontrará como formulação dessa integração, o higienismo dos séculos XVIII e XIX. Essa aproximação é importante, uma vez que a epidemiologia não se restringirá a uma classificação das doenças, mas compreenderá uma classificação da relação das doenças com o espaço e com os grupos que ocupam certos espaços. Assim, permitirá uma formulação da doença na relação com o corpo que a apresenta, um corpo não apenas individual, mas um corpo social:

seu suporte não é a percepção do doente em sua singularidade, é uma consciência coletiva de todas as informações que se cruzam, crescendo em uma ramagem complexa e sempre abundante, ampliando finalmente até as dimensões de uma história, de uma geografia, de um Estado. (Foucault, 1994, p. 32).

A aproximação entre a doença e a história, entre a geografia e o Estado, permitirá que no século XIX, a medicina se aproxime mais da normalidade do que da saúde, sendo que a ciência da vida tornar-se-á uma ciência do normal e do patológico. Conforme aponta discussão de Canguilhem (2012), essas duas dimensões tornamse fundamentais para o conhecimento e prática médica, sobretudo, para focalizar o normal em dois eixos: regularidade estatística e padrão. Isso significa que, inclusive, o patológico pode assumir uma dimensão de normalidade, quando equalizado à médias ou padrões de um grupo ou população. Porém, o autor salienta que o termo normal não é absoluto, justamente por sua condição de relação a outros termos, tais como a média e o padrão. Desse modo, tanto o normal quanto o patológico dependem de relações, ou seja, de outros termos que os qualificam como tais: "todo indivíduo anômalo (portador de anomalias), isto é, aberrante em relação a um tipo específico estatisticamente definido." (Canguilhem, 2012, p. 176).

A relação entre o normal e o patológico constitui uma condição de possibilidade para a construção de perfis epidemiológicos. O perfil epidemiológico refere-se à caracterização de indivíduos, grupos, população, por meio de dimensões epidêmicas, geográficas, sociais, circunscritas tanto pela normalidade quanto pela patologia. Ou seja, o perfil epidemiológico constituirá tanto aquilo que é normal e o que se opõe à norma no âmbito sociológico, quanto o que é normal e o que se opõe à norma no âmbito do patológico. O perfil epidemiológico tornar-se-á, a partir da epidemiologia do século XIX, uma forma de produção de conhecimento que marcará a ciência da vida como uma ciência de Estado. Isso será um eixo fundamental para as estratégias de governo da população: traçar uma linha que permita a organização de um conjunto de 
elementos que aparecem dispersos no tecido social. O perfil epidemiológico permite um conhecimento sobre aquilo que se governa; não se trata apenas de uma classificação de doenças, mas, sobretudo, da distribuição em um espaço das regularidades e irregularidades, de modo que as características epidêmicas e endêmicas criam séries com aspectos sanitários, de renda, de escolaridade, de faixa etária. Isso porque haverá uma aproximação da ciência estatística e se constituirá um cruzamento sistematizado entre elementos heterogêneos, o que possibilita fazer surgir interligações e dependências.

O perfil epidemiológico, então, cria condições para que se constitua a população, ao mesmo tempo de forma homogênea e heterogênea. O perfil dará à população a possibilidade de certas singularidades/ irregularidades em termos de desenhos específicos dos grupos que a constituem. A epidemiologia, então, permitirá que a população seja tomada a partir das regularidades, ou seja, da relação entre o normal e o patológico.

Frente a isso, a ciência da vida aproximar-se-á do Estado e da ciência estatística. Esses três elementos articulados permitem, além do aparecimento do problema da população, como uma realidade com seus fenômenos específicos, o surgimento de estratégias de governo voltadas para a própria população, sendo que "gerir a população quer dizer geri-la igualmente em profundidade, em fineza e no detalhe" (Foucault, 2003, p. 302). A profundidade, a fineza e o detalhe tornam o perfil epidemiológico uma estratégia privilegiada de Políticas de Saúde que atualmente, para Monken e Barcellos (2005), se faz também por uma possibilidade de articulação de conceitos das ciências humanas a procedimentos epidemiológicos e geográficos, permitindo avançar os mecanismos de sistematização e planejamento de indicadores de saúde e ações de saúde.

Desse modo, esses três elementos — vida, Estado e estatística- constituem, a partir do século XVIII e, sobretudo, no século XIX, uma governamentalização do Estado, conjuntamente com a constituição de uma forma de saber nomeada de economia política, que se formula por esses elementos também. A governamentalização do Estado tornar-se-á uma modalidade de gestão da vida, da vida da população, encontrando, entre outros elementos, a formulação de políticas para a administração dessa vida.

A governamentalidade não é uma arte de governo específica do Estado, mas constituirá, justamente, a possibilidade do Estado como um gestor privilegiado da vida da população. Isso cria uma série em que se alinham Estado-governo-população. As formas de governo, nesse caso, voltam-se para os fenômenos da vida, ou seja, são formas de biopolíticas. Dessa maneira, a governamentalidade biopolítica envolve tanto equipamentos, procedimentos e táticas quanto tipos de saberes específicos, em que os dispositivos de segurança são instrumentos técnicos essenciais. Frente a isso, as Políticas de Saúde possibilitam um arranjo entre saberes específicos (ciências da vida, geografia, história, estatística, ciências humanas) e dispositivos de segurança, na medida em que criam tecnologias/procedimentos/táticas voltados para a gestão da vida de uma população.

A governamentalidade terá como foco privilegiado a vida da população, e isso engendra-se a partir de uma estratégia biopolítica, ou seja, de um poder que se volta para a vida e para o fazer viver. Nesse caso, a governamentalidade tem como estratégia de gestão, a biopolítica. A biopolítica é um modo de gerir da governamentalidade, abarcando os fenômenos da população (nascimentos, óbitos, epidemias, endemias, saneamentos/moradias, etc.).

Assim, o perfil epidemiológico, como foi apontado, permite às Políticas operarem no interior da população, tanto na direção do conjunto quanto na das singularidades. O perfil epidemiológico torna-se um modo de circunscrição de territórios e de modos de acesso. 


\section{Territórios e modos de acesso}

O conceito de território é circunscrito pelas Políticas de Saúde, seja no sentido de referência à nação - "Art. $1^{\circ}$. Esta Lei regula em todo o território nacional” (Brasil, 1990, p. 1), seja no sentido de lócus privilegiado do desenvolvimento das ações de saúde,

desenvolvida[s] por meio do exercício de práticas gerenciais e sanitárias democráticas e participativas, sob forma de trabalho em equipe, dirigidas a populações de territórios bem delimitados, pelas quais assume a responsabilidade sanitária, considerando a dinamicidade existente no território em que vivem essas populações (Pacto pela Saúde, 2006, p. 10).

Essa dupla vetorização do território conforma dois elementos importantes: o de nação e o de espaço de ações. Ela permite que a Política de Saúde seja uma política que regula, no espaço geográfico da nação, as ações de saúde. Desse modo, opera-se na definição de uma geografia e de ações que se voltam para essa geografia, de maneira a tornar o território um espaço físico de circulação de ações. Essa obviedade é importante de ser colocada em análise, uma vez que esses elementos que vetorizam o território e que, portanto, o constituem como o espaço onde ações em saúde se desenvolvem, acabam justamente por tornarem-se condições de possibilidade para uma analítica do exercício de poder, no sentido de práticas de governo da vida, sendo que as noções de nação e de ações de saúde se tornam fundamentais para essa compreensão, no que diz respeito à temática deste artigo. Além disso, marca um espaço de inflexão para a Psicologia, na medida em que permite uma problematização do território, não apenas como meio ambiente, no qual a vida se desenrola e que influenciaria o humano em uma relação de causa-efeito. A inflexão torna possível considerar o território como espaço de tensão, no qual não se trata apenas de meio e localização, e sim de relações de poder e verdade que produzem subjetividades. Isso permite tanto uma complexificação da disciplina psicológica, quanto um outro modo da mesma articular-se às políticas públicas de saúde.

A noção de nação para pensar o território é importante quando esta se refere a um campo social que se constitui por uma população e por uma forma de organização social, tomada como uma sociedade. A nação conjuga elementos heterogêneos circunscritos em um espaço e torna-os homogêneos em termos de pertencimento, ou seja, eles são parte de uma nação. A nação terá a preocupação de definir suas fronteiras geográficas, mas, especialmente a partir do século XVIII, de definir suas diversidades internas (Foucault, 2008). Nesse caso, a nação traça uma possibilidade de investimento sobre si mesma, principalmente de investimento na população que a constitui em termos de espaço nacional. Desse modo, território acaba por tornar-se um elemento fundamental para o governo daquilo que está dentro e não propriamente daquilo que o rodeia. A delimitação de um território nacional permite estabelecer o espaço onde se estendem as possibilidades de regulação da população:

integração, em nível executivo, das ações de saúde, meio ambiente e saneamento básico; conjugação dos recursos financeiros, tecnológicos, materiais e humanos da União, dos Estados, do Distrito Federal e dos Municípios, na prestação de serviços de assistência à saúde da população

(Brasil, art.7, incisos X e XI, p. 5).

Nação e ações de saúde permitem considerar, no âmbito das Políticas de Saúde, o território como um espaço geográfico onde a vida se desenrola, tanto em seus aspectos fixos quanto em seus fluxos (modos de circulação e uso dos aspectos físicos), onde se congregam elementos humanos e não-humanos. Monken e Barcellos (2005) consideram que as ações em saúde são dirigidas à vida cotidiana, que se apresenta nos territórios, o que permite 
que as práticas em saúde se aproximem efetivamente dos problemas e necessidades sociais de saúde. Assim, considera-se que a Atenção Básica à Saúde é a porta de entrada no sistema de saúde, como também estratégia privilegiada das ações de saúde, já que objetiva

possibilitar o acesso universal e contínuo a serviços de saúde de qualidade e resolutivos, caracterizados como a porta de entrada preferencial do sistema de saúde, com território adscrito de forma a permitir o planejamento e a programação descentralizada, e em consonância com o princípio da equidade (Pacto pela Saúde, 2006, p. 11).

O território adscrito e as ações de saúde permitem o investimento da nação na população, a partir de uma estratégia geopolítica, ou seja, tanto do elemento fixo - espaço onde a população se encontra — quanto das ações — formas de vida da população.

Como o território será composto por sua geografia e pela circulação nessa geografia, ele acaba por tornar-se um domínio privilegiado das ações e estratégias de regulação, pois será sobre ele e a partir dele que um conjunto de práticas em saúde se constituirá. Domínio não como algo concreto de pertencimento, como uma instituição, por exemplo, mas como um campo estratégico, sobre o qual um conjunto de ações se desenvolve. Até aqui, não haveria propriamente o que se interrogar, porém, é importante considerar que, como campo estratégico, o território é tanto o espaço constituído quanto o espaço que constitui as ações. Ou seja, é o espaço para onde se voltam as ações, ao mesmo tempo em que é uma estratégia que define as ações que nele serão desenvolvidas, demarcando regiões para as quais as ações se dirigem em suas especificidades, de modo a tornar-se um vetor de produção de subjetividades.

O avizinhamento das ações com o território é colocado pelas Políticas de Saúde a partir de uma estratégia que articula epidemiologia e prestação de serviços:

Art. 47. O Ministério da Saúde, em articulação com os níveis estaduais e municipais do Sistema Único de Saúde-SUs, organizará, no prazo de 2(dois) anos, um sistema nacional de informações em saúde, integrado em todo o território nacional, abrangendo questões epidemiológicas e de prestação de serviços (Brasil, 1990, p. 18).

Torna-se possível uma gestão da saúde a partir de elementos que constituem uma arquitetura de indicadores e ações voltadas para esses indicadores em um território, que é circunscrito e que circunscreve, segundo o qual a epidemiologia se tornará necessária para “o estabelecimento de prioridades, a alocação de recursos e a orientação programática" (Brasil, 1990, p. 4, art. 7 VII). Ao costurarem-se esses elementos - território, epidemiologia, ações - definem-se as formas de acesso por meio da alocação de recursos e orientação programática. Para a Psicologia, no campo da saúde, passa a ser importante considerar que acesso, alocação de recursos e orientação programática não se tratam apenas de medidas administrativas, mas, sobretudo, que essa forma de administração tem efeitos múltiplos nos modos de viver e habitar um espaço. Isso significa apontar uma indistinção entre o sujeito para o qual se voltam essas ações e a própria forma como esse sujeito irá se constituir, ou seja, se subjetivar. Não se trata especificamente de ponderar uma mera aplicação de recursos e tecnologias, mas de produção de subjetividades.

A importância que assumem na Política de Saúde os conceitos de território e epidemiologia, diz respeito às formas de acesso, porque é por meio delas que se torna possível definir a própria população. Nesse caso, a governamentalidade como modalidade de gestão da vida opera na produção de uma forma de conhecer, que também constitui aquilo mesmo que se dá a conhecer dentro desse espaço que é o território. O território e a 
epidemiologia permitem, assim, a constituição da população em termos de necessidades e problemas de saúde, fabricados pela própria estratégia de territorialização e caracterização epidemiológica que constitui as formas de acesso. Essa aproximação do território com a epidemiologia faz-se por práticas divisoras internas e externas - diferentes sujeitos dentro de um grupo, diferentes grupos dentro de uma população-, que transformam modalidades de vida em perfis e territórios, ou seja, em formas de subjetividade. Dessa forma, a universalidade do acesso, tal qual prevista na legislação, operacionaliza-se por demandas criadas pelo território epidemiológico, como práticas divisoras, o que permite absorver elementos heterogêneos e torná-los homogêneos, mediante o que Deleuze e Parnet (1998) consideram como formas de territorialização. O deslocamento do heterogêneo para o homogêneo não é um processo unilateral, só de um para o outro.

Em se tratando da relação entre território e epidemiologia, as singularidades/irregularidades migram para um campo de regularidades quando se tornam perfis, mesmo que de anormalidades, porém característicos da normalidade de um grupo (por exemplo, a diabetes e a hipertensão são consideradas como anormalidades orgânicas, porém normalidades sociológicas de certos grupos da população, como os idosos). Mais ainda, migram do homogêneo para o heterogêneo quando, ao instituir-se uma Política como a de Atenção Integral à Saúde, esta se esquadrinha por subsistemas de saúde, em razão das especificidades e irregularidades da população (Saúde do Idoso, Saúde da Mulher, Saúde do Homem, Saúde dos Povos Indígenas, etc.). Porém, esse jogo é de capturas e circunscrições, ou seja, territorializações, pois o que o torna possível são as distintas práticas que territorializam modalidades e formas de viver em focos e perfis a serem governados e que estabelecem as condições de acesso.

Para Deleuze e Parnet (1998), a territorialização é um processo que compõe segmentaridades determinadas, ou seja, define identidades a segmentos, colocando-os em uma linha de serialização que opera por procedimentos binários e diacrônicos. Dito de outro modo, a territorialização sedimenta modalidades e formas de viver em campos identitários e organiza-os de maneira a comporem linearidades comparativas ou consecutivas: homem-mulher, branco-negro, criança-jovem-adulto-idoso, família-trabalho-aposentadoria. Os processos de territorialização são máquinas que nos cortam “em toda espécie de sentidos" (p. 149), e a relação que se estabelece com as formas de governo da vida é que as segmentaridades implicam dispositivos de poder, na medida em que esses dispositivos permitem que os códigos se fixem às condutas que lhes correspondem.

O acesso, assim, torna-se possível nas estratégias de territorialização, isto é, a articulação entre território e epidemiologia, como estratégia de governamentalidade, será um processo de territorialização da população e das formas de acesso dessa população às Políticas de Saúde a partir de procedimentos que compõem segmentaridades. $\mathrm{O}$ acesso define-se como ações que investem na população e ações da população em direção a esses investimentos - um dispositivo que faz com que códigos se fixem às condutas, e que as condutas se fixem aos códigos, tornando possível o governo de uma população.

Nessa linha de argumentação, Rose (2014) aponta que o governo da população envolve um conjunto de heterogêneos mecanismos de administração da vida, que se constituem por modalidades de exercícios de poder: "o território traçado pela multiplicidade de regimes, sonhos, cálculos e estratégias para a conduta da conduta" (p. 41). Assim, as formas de governo da população territorializam-se por modos de exercício de poder que focalizam a conduta da conduta. Se considerarmos, então, conforme escrito anteriormente, que a Política de Saúde conforma um território de investimentos e também define aquilo que habita/ circula em um território a partir de uma estratégia 
epidemiológica, é possível compreender que esses mecanismos de condução de condutas tornam o acesso uma estratégia para a própria condução. A territorialização, então, como um exercício de governo das condutas, constitui-se por uma estratégia, que é a do acesso. O acesso permite o ingresso no sistema de saúde e o ingresso da saúde no território por meio de mecanismos que circunscrevem e produzem as características da população que acessa - perfis epidemiológicos. Nesse caso, o perfil epidemiológico marcará a conduta a ser conduzida e a conduta que permite acessar, de modo que a territorialização se torna um mecanismo para acessar o próprio território e para o acesso ao investimento nesse território.

A relação de reciprocidade dá-se a partir da efetividade dos modos de condução da conduta que permitem o acesso. $\mathrm{O}$ acesso é universal, na medida em que se entende que o que o torna possível é um governo das condutas e um perfil epidemiológico que as circunscreve, ou seja, é a forma de condução da conduta/perfil epidemiológico, que permite a universalidade do acesso: é preciso ser mulher adulta para acessar um programa de saúde da mulher; é preciso ser diabético e hipertenso para acessar um programa de saúde do idoso; é preciso ser residente fixo para acessar as Unidades Básicas de Saúde. Esse "ser" caracteriza-se por modalidades de conduta que estabelecem formas de subjetivação, como o "ser mulher", o "ser idoso", o "ser morador". A condução da conduta refere-se ao modo como os sujeitos conduzem a si mesmos, a partir de um campo que os constitui, ou seja, de formas de subjetivação produzidas por processos de territorialização, como escrito anteriormente.

$\mathrm{O}$ acesso determina o modo de ser da conduta porque é ela que permite o ingresso; ao determinar o modo de ser da conduta, também definirá a conduta que poderá acessar. Portanto, o acesso compõe tanto a possibilidade de caracterização da população, ou seja, de um perfil epidemiológico, pois é por ele que se aproximam estratégias de governo da população e quem é essa população, quanto à forma como o perfil epidemiológico permitirá o acesso, na medida em que, ao conduzir-se de determinados modos, a população passa a ter tal possibilidade.

É nesse domínio — ações que investem e ações que permitem acessar os investimentos - que se pode compreender, a partir da noção de relações de poder, que um jogo agonístico se constitui pelo acesso. Trata-se de um jogo agonístico, porque os modos de acesso que se constituem no território, se definem por elementos fixos, mas também por fluxos, e essa dimensão dos fluxos apresentará permanentemente lutas transversais, ou seja, elementos heterogêneos que aparecem no território e o fazem vacilar.

\section{Negociação, enfrentamentos, tecnologias novas de acesso}

A relação entre territorialização, perfil epidemiológico e acesso, é importante para a Psicologia, principalmente porque historicamente a disciplina constitui-se como um campo de homogeinização social, apoiado entre outros vetores, em uma perspectiva médica que toma como fio condutor de compreensão e intervenção o normal. Desse modo, quando a Psicologia se volta para a problematização do próprio normal, por meio de perspectivas críticas, isso permite uma modificação da disciplina, que possibilitará outros modos de pensar e intervir, ou seja, um outro jogo político no campo das políticas públicas, por exemplo. Esse outro jogo político passa a considerar que a heterogeneidade pode ser um modo de contestação das formas de homogeinização, de produção do normal, que se constituem pelas territorializações. A Psicologia, assim, entraria como parte de estratégias de produção de diferença e não mais de normalidades.

Desse modo, fazer vacilar é o que permite algumas questões neste texto, pois se trata daqueles elementos que colocam em análise os modos de funcionamento e produção das ações em saúde. 
O exercício de colocar em análise refere-se a uma possibilidade de considerar que, mesmo em se tratando de governo das condutas, há de se pensar também se as condutas precisam sempre ser governadas (Lagasnerie, 2013). Entende-se que, a relação entre acesso e os fluxos, não deve apontar para que outras estratégias devam ser criadas para a captura desses fluxos, mas como os fluxos nos ajudam a pensar sobre o que se territorializa e como esse processo se dá a partir de mecanismos políticos de governo da vida. Desse modo, o acesso e a forma como se articula ao território podem ser tomados, no campo da saúde, como "um dispositivo que devemos tomar como objeto: é ele que convém examinar, retrabalhar, reformular" (Lagasnerie, 2013, p. 15). Considerar a articulação entre acesso e território como um dispositivo, a partir daquilo que o faz vacilar, seria um exercício de pensar/trabalhar com dispositivos experimentais que permitam justamente pensar de outras formas.

Para a Psicologia, no campo das políticas públicas, é importante focalizar a singularidade como dispositivo de produção de diferença. Trata-se de um modo de torção do singular que o migra de uma dimensão da anormalidade/patologia para o de diferença. Neste caso, ao entrar no campo da saúde, a Psicologia abre-se não para um exercício de captura da diferença pela patologia, e sim, da diferença como exercício de contestação de modos de governo da vida. A produção de diferença opera como política de tensionamento do perfil epidemiológico e das territorializações, assinalando outras possibilidades de subjetivação. Isso implica considerar no campo da saúde, não apenas o modo de intervir, mas como certos elementos, certas formas de viver permitem modificar as próprias intervenções.

Mendes e Cavendon (2012) apontam a importância de considerarem-se, nos processos de análise, as práticas microbianas - aqueles elementos singulares, microscópicos, plurais, que se apresentam nos territórios a partir de planos de contestação das modalidades de produção e organização social.
Segundo os autores, seria interessante percorrer, de modo etnográfico, enunciações pedestres e retóricas ambulantes. Os dispositivos experimentais em saúde poderiam agenciar-se, não por perfis epidemiológicos, mas por enunciações pedestres e retóricas ambulantes, que se fazem como elementos singulares, microbianos, e não normalidades, anormalidades e patologias, que condicionam o acesso, no cotidiano dos territórios.

Fazer vacilar coloca em análise o dispositivo e torna-o experimental, porque abre um campo de possibilidades que interrogam aquilo que produzimos como grades de inteligibilidade na saúde. As reformas na saúde e as lutas pela implantação de um sistema de saúde implicado no direito à vida, não percorrem de maneira marginal as formas de governo da vida; são outras modalidades de exercícios de poder, que têm como foco a vida e como estratégia também a condução das condutas. Porém, o dispositivo também pode ser considerado como um elemento analisador daquilo que se produz, como formas de governo da vida.

Desse modo, a Psicologia pode contribuir para a produção de dispositivos experimentais de contestação, a partir de formas de singularização dos modos de viver. A singularização aqui diz respeito a todas as formas de viver que se apresentam como possibilidades de não assujeitamento. Um não assujeitamento a certas modalidades de condutas e perfis epidemiológicos. A Psicologia intervém não para o exercício do mesmo, ou seja, para o enquadramento e esquadrinhamento de sujeitos e modos de viver, mas para a potencialização e invenção da diferença. Trata-se de uma orientação para planos de bifurcação, que constituem outras possibilidades de viver, sem tomar como vetor exclusivo a relação entre normal/patológico.

Isso porque o governo das condutas, de diferentes modos, opera com base em discursos totalizantes, o que se vê pelo perfil epidemiológico. Mesmo que a Política de Saúde seja, no Brasil, uma estratégia de crítica a procedimentos totalizantes, ao basear-se em elementos que totalizam, como a 
necessidade de perfis epidemiológicos para a definição de estratégias e modos de acesso à saúde, acaba por forjar territórios, sendo estes formas de totalização. Quando as formas de governo das condutas se encontram com a vida, não apenas aparecem os assujeitamentos a essas formas, mas também há uma proliferação de lutas, como já apontava Foucault (2005) quando da análise da formação das nações.

Nesse caso, o fazer vacilar indica a não submissão, a profusão de ofensivas dispersas e descontínuas, que justamente indicam a desestabilização dos territórios, ou seja, torna-se fundamental que a Psicologia entre na esteira de intervenções de desestabilização de territórios também, como um exercício político da disciplina. Essas formas de desestabilizar-se do familiar encontram-se naquelas modalidades de viver e de conduta que extrapolam os protocolos. Como responder às jovens que engravidam porque querem? Como responder ao morador de rua que não tem residência fixa porque justamente mora na rua? Como responder ao idoso que vai à Unidade de Saúde porque se sente solitário? A problemática do protocolo está justamente em tornar essas demandas em problemas, portanto, carecendo de respostas. A questão também não é não considerar que aquele que mora na rua não deva ter acesso a uma residência, nem de se pensar o que fazer com a gravidez na juventude ou com a solidão. Mas por que a resposta tem que ser como uma forma de condução da conduta definida pelo acesso, circunscrito a um perfil epidemiológico? Por que essas vidas precisam ser reguladas, novamente a partir de um exercício de territorialização?O encontro com a vida traz para a Psicologia no campo da saúde, mais do que necessidades de captura da vida, aquilo que supostamente tomamos como familiar: a importância da definição de perfis para o estabelecimento de estratégias. A vida, então, apresentar-se-á ao acesso, não só como subsumível a ele, mas também como aquilo que o contesta, contestando, inclusive, o que na saúde se considera como o melhor para o outro, de modo a tornar-se uma arte de não se ser governado em demasia (Foucault, 1990). De acordo com a análise de Lagasmerie (2013) sobre o pensamento foucaultiano, a crítica das ideias gerais, das teorias totalizantes ou pensamentos do fundamento constitui o ponto de partida da invenção de uma nova política, que se definirá como uma política das singularidades, uma política de acompanhamento e apoio às lutas múltiplas e aos combates setoriais (p. 109). Aos processos de totalização, forjam-se lutas parciais e locais, em que as categorias de análise/investimento não são mais suficientes, porque são coercitivas.

\section{Referências}

Brasil. Lei $n^{\circ}$. 8080, de 19 de setembro de 1990. Lei Orgânica da Saúde. Dispõe sobre as condições para a promoção, proteção e recuperação da saúde, a organização e o funcionamento dos serviços correspondentes e dá outras providências. Diário oficial da união. Brasília, D.F., p. 005346, set. 1990. Seção 1. Recuperado de http://portal.saude.gov.br/portal/arquivos/pdf/ lei8080.pdf

Canguilhem, G. (2012). O conhecimento da vida. Rio de Janeiro: Forense Universitária.

Czeresnia, D. (2001). Constituição epidêmica: velho e novo nas teorias e práticas da epidemiologia. História, Ciências, Saúde-Manguinhos, 8(2), 341-356.

Deleuze, G., \& Parnet, C. (1998). Diálogos. São Paulo: Editora Escuta.

Foucault, M. (1994). O nascimento da clínica. Rio de Janeiro: Forense Universitária.

Foucault, M. (2003). Ditos \& Escritos IV: estratégia Poder-Saber. Rio de Janeiro: forense Universitária.

Foucault, M. (2005) Em defesa da sociedade. São Paulo: Martins Fontes.

Foucault, M. (2008). Segurança, território, população. São Paulo: Martins Fontes. 
Foucault, M. (1990) O que é a crítica? [Crítica e Aufklärung]. Espaço Michel Foucault. Em M. Foucault. Qu'est-ce que la critique? Critique et Aufklärung. Bulletin de la Société française de philosophie, (82(2), pp. 35-63), avr/juin 1990. (Conferência proferida em 27 de maio de 1978). Tradução de Gabriela Lafetá Borges e revisão de wanderson flor do nascimento. Recuperado de http://michel-foucault.weebly.com/textos.html Lagasnerie, G. (2013). A última lição de Michel Foucault. São Paulo: Três Estrelas.

Mendes, L., \& Cavendon, N. R. (jan./jun. 2012). A atividade de camelô como prática urbana no contexto das cidades. Urbe: Revista Brasileira de Gestão Urbana (Brazilian Journal of Urban Management), 4(1), 123-140.

Monken, M., \& Barcellos, C. (may/june 2005). Vigilância em saúde e território utilizado: possibilidades teóricas e metodológicas. Cadernos de Saúde Pública, 21(3).

Rose, N.. (2014). Poder e subjetividade: história crítica e psicologia. Em. Guareschi, Neuza Maria de Fátima, Azambuja, Marcos Adegas \& Hünning, Simone Maria. Foucault e a Psicologia na produção de conhecimento. Porto Alegre: EDIPUCRS.

\section{Recebido: setembro 15, 2015 Aprovado: agosto 3, 2016}


